\title{
Usability of Nasal Glucagon Device: Partially Randomized Caregiver and Third-Party User Experience Trial with Simulated Administration at a Japanese Site
}

Toshihiko Aranishi (D) Y Yukiko Nagai - Yasushi Takita •

Shuyu Zhang $\cdot$ Rimei Nishimura

Received: July 11, 2019 / Published online: November 4, 2019

(C) Eli Lilly and Company 2019

\section{ABSTRACT}

Introduction: Glucagon is the only approved medicine for severe hypoglycemia available for caregivers of people with diabetes. Nasal glucagon (NG) was recently approved in the USA as a needle-free, ready-to-use alternative to

Enhanced Digital Features To view enhanced digital features for this article go to https://doi.org/10.6084/ m9.figshare.9784958.

Electronic supplementary material The online version of this article (https://doi.org/10.1007/s13300019-00711-1) contains supplementary material, which is available to authorized users.

T. Aranishi $(\bowtie)$

Health Outcomes/Health Technology Assessment/ Real World Evidence, Medicines Development UnitJapan, Eli Lilly Japan K.K., Kobe, Japan

e-mail: aranishi_toshihiko@lilly.com

Y. Nagai

Diabetes Products, Medicines Development Unit-

Japan, Eli Lilly Japan K.K., Kobe, Japan

Y. Takita

Statistical Science, Medicines Development Unit-

Japan, Eli Lilly Japan K.K., Kobe, Japan

S. Zhang

Global Statistics and Science, Eli Lilly and Company, Indianapolis, IN, USA

R. Nishimura

Division of Diabetes, Metabolism and

Endocrinology, Department of Internal Medicine,

The Jikei University Hospital, Tokyo, Japan injectable glucagon. This simulated user experience study in Japan compared NG and intramuscular glucagon (IMG) administration by caregivers, and NG administration by untrained third parties.

Methods: This was an open-label, single-center, partially randomized crossover, simulated user experience trial conducted in Japan (October 2018 to December 2018). Caregivers who live with and care for a relative with diabetes were randomized (1:1, stratified by patient diabetes type 1 or 2 ) to one of two simulated administration sequences (group 1: NG then IMG; group 2: IMG then NG). Caregivers received training on each device 2 weeks before simulated administration of the device. Third parties received no training and only conducted simulated NG administration. Outcome measures included the percentage of successful administrations (based on critical step completion and dose; primary outcome), time to complete administration, and user satisfaction/ preferences.

Results: In caregivers $(N=19)$, the percentage of successful administrations was greater $(89.5 \%$ vs $26.3 \%, P<0.001$ ) and mean time to complete administration was shorter (23.9 vs 207.3 s, $P<0.001)$ with NG than with IMG. In third parties $(N=20)$, 95\% of NG administration attempts were successful (mean time to complete administration, $55.5 \mathrm{~s}$ ). All caregivers and $80 \%$ of third parties reported that the NG device was easy to use. All caregivers and $70 \%$ of 
third parties were confident and willing to use the device in a real emergency, and more than $80 \%$ of caregivers preferred the NG device to IMG.

Conclusion: This simulated user experience study confirmed that glucagon administration using a nasal delivery device was quicker, easier, and had a higher success rate than intramuscular administration in Japan, where the glucagon injection kit is not available.

Funding: Eli Lilly.

Plain Language Summary: Plain language summary available for this article.

Keywords: Administration; Caregivers; Diabetes mellitus; Glucagon; Hypoglycemia; Intranasal; Intranasal administration; Patient preference

\section{Key Summary Points}

Nasal glucagon (NG) is currently being developed as a needle-free, ready-to-use alternative to injectable glucagon for use by caregivers for the treatment of severe hypoglycemia in patients with diabetes.

In this simulated user experience trial conducted in Japan, we hypothesized that the NG device would be faster and more accurate when used by caregivers, compared with intramuscular glucagon (IMG) administration.

We also hypothesized that the NG device could be used successfully by untrained third parties.

Both the speed and the rate of successful glucagon administration were significantly greater with the NG device than with IMG injection, and caregivers strongly preferred using the NG device over IMG.

The availability of a nasal glucagon delivery device would likely increase the willingness of patients and their caregivers to keep glucagon with them and administer it in the event of a severe hypoglycemic episode.

\section{PLAIN LANGUAGE SUMMARY}

Glucagon is a drug used to treat people with diabetes when they have very low blood sugar levels (severe hypoglycemia), which can lead to unconsciousness. However, injection of glucagon into muscle (e.g., in the hip) using a needle and syringe takes multiple steps and can be confusing and difficult for caregivers of patients, especially in an emergency. In this study, we tested a new way to give glucagon using a needle-free, ready-to-use device that releases drug into a patient's nose. The study was done in Japan and involved relatives of people with diabetes (caregivers) who were trained on injecting glucagon into muscle and on using the nasal device before trying it on a manikin 2 weeks later. The study also included "third parties" who did not have a relative with diabetes and who were tested on the nasal device without any training. In the caregiver group, the nasal device was quicker, easier, and had a higher success rate (delivering an effective dose) than injection. Third parties could also use the nasal device successfully. In a questionnaire, all caregivers and most third parties commented that the nasal device was easy to use, and they were confident and willing to use it in an emergency. When asked to compare the two methods, caregivers strongly preferred the nasal device to injections. These results suggest that a nasal device might make people with diabetes more likely to keep glucagon with them and their caregivers more likely to use it when needed.

\section{INTRODUCTION}

Severe hypoglycemia is a serious condition that can occur in patients with type 1 (T1D) or type 2 diabetes (T2D). By definition, hypoglycemia is considered severe when the patient cannot selftreat through carbohydrate ingestion and must rely on others to provide assistance [1]. If not treated, severe hypoglycemia can result in reduced cognitive function, unconsciousness, seizures, and, if prolonged, coma and death $[2,3]$. Moreover, fear of hypoglycemia adversely 
affects quality of life for the majority of patients and their caregivers [4-6].

Glucagon is the only approved medicine for severe hypoglycemia available for use $[1,7]$ by caregivers in Japan. Currently available glucagon must be administered by intramuscular injection for the treatment of hypoglycemia, which involves multiple steps that can be confusing, as well as daunting, to caregivers. In a simulation study, $69 \%$ of parents of children or teenagers with diabetes had difficulty handling the components required to prepare for glucagon injection, and often injected less than a full dose [8]. Although training in intramuscular glucagon (IMG) administration is recommended [7], caregivers do not always receive instructions and few have hands-on training [8]. Glucagon for intramuscular injection is available in kit form in many countries; however, this is not the case in Japan. Instead, glucagon powder, diluent, syringes, and needles required for IMG administration are only available as separate components. This increases the complexity of preparing glucagon for intramuscular injection and may also increase the chance of mistakenly administering other drugs such as insulin in an emergency. The reluctance of caregivers to inject glucagon may contribute to the low possession rate for glucagon in Japan [9].

Nasal glucagon (NG) has recently been approved in the USA, and is under consideration in other countries, for emergency treatment of severe hypoglycemia as a needle-free, ready-to-use alternative to IMG (Fig. 1; video in electronic supplementary material). Nasal glucagon has been shown to have similar efficacy to IMG for the treatment of severe hypoglycemia in adults, children, and adolescents with T1D, both when delivered by health care professionals in controlled trials [10-12] and when delivered by caregivers in non-medical settings [10-12]. Further, users of the NG device in real-world situations reported that it was easy to use $[13,14]$. A previous study conducted in Canada, in which caregivers and third parties simulated glucagon administration using a manikin, indicated that the full dose of glucagon was achieved more frequently with NG than with IMG [15]. The time and skill required

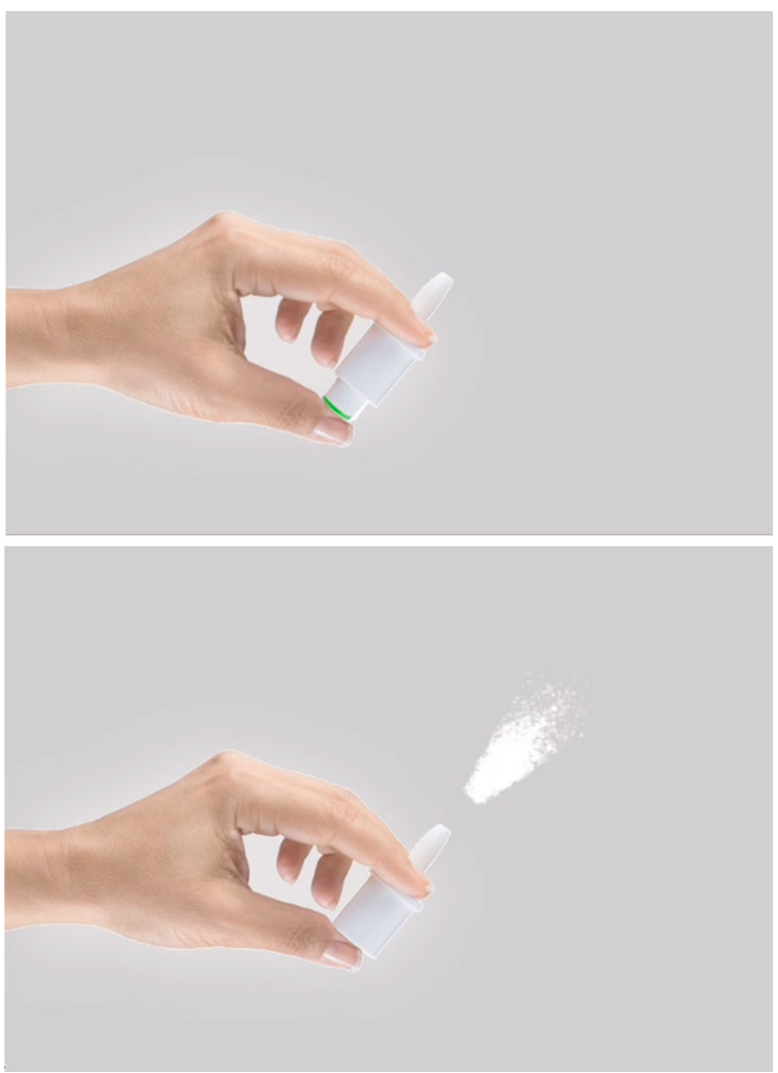

Fig. 1 Nasal glucagon device. Image copyright of Eli Lilly and Company

to inject IMG, and the relative improvements provided by a nasal delivery alternative, may be even greater in Japan, where the glucagon kit is not available.

The aim of the current simulation study conducted in Japan was to compare NG and IMG administration by caregivers, and to assess NG administration by untrained third parties. We hypothesized that, compared with IMG administration, the NG device would be faster, have a higher rate of successful administration, and be preferred by users.

\section{METHODS}

\section{Study Design}

This was an open-label, single-center, partially randomized crossover, simulated user experience trial conducted between October and 
December 2018 (first participant enrolled October 27, 2018; last participant completed December 22, 2018). The protocol was approved by the ethics review committee of the study site (PS Clinic), and the study was conducted in accordance with the principles of the Declaration of Helsinki, Ethical Guidelines for Medical and Health Research Involving Human Subjects in Japan [16], and all applicable laws and regulations. As this was a non-interventional trial, submission to a clinical trial registry was not required. All participants provided informed consent before the start of the study.

\section{Study Population}

The trial enrolled two types of participants: caregivers and third parties. Caregivers were defined as people who live with and care for a relative with diabetes and were assumed to have knowledge of the therapeutic effect of glucagon for severe hypoglycemia. Third parties were defined as people who do not live with or care for a person with diabetes and who have not been trained to administer glucagon. Men or women aged 20 years or more were eligible for enrollment. Caregivers were eligible for inclusion if they were first- or second-degree relatives who lived with and cared for a patient with T1D or T2D (if T2D, the patient must be treated with insulin) who agreed not to learn how to administer glucagon during the study, except as part of the trial. Third parties were eligible for inclusion if they did not live with and care for a relative with diabetes. For both caregiver and third-party cohorts, people who were diagnosed with T1D or T2D, had previous experience injecting glucagon, were a health care provider or pharmaceutical company employee, or who were judged by an investigator as having been sufficiently trained or educated to enable them to perform the simulated administration with ease were excluded.

\section{Study Protocol}

\section{Caregivers}

At visit 1, enrolled caregivers were randomized [1:1, using a randomization table and stratified by patient diabetes type (T1D or T2D)] to one of two simulated administration sequences: group 1 simulated glucagon administration using the NG device first, followed by IMG, and group 2 simulated IMG administration first, followed by the NG device. Randomizing the sequence of glucagon administration methods in the two groups was intended to minimize any potential learning effects between each administration device simulation. After randomization, caregivers were individually trained on their first device (NG or IMG). Health care providers (e.g., nurse or pharmacist) explained the steps involved in using the device and demonstrated its use. The order of critical steps was also explained. Caregivers did not perform simulated administration during the training session but could ask questions about administration. There was no time limit on the training session. At visit 2 ( $14 \pm 2$ days later), caregivers performed simulated administration using their first assigned device. On the same day, caregivers were trained on their second device, as above. At visit 3 (14 \pm 2 days after visit 2), caregivers performed simulated administration using their second assigned device.

\section{Third Parties}

Third parties received information about the therapeutic effect of glucagon on hypoglycemia, but did not receive training on use of the device before simulated administration. At visit 1 , enrolled third parties performed simulated administration using the NG device.

\section{Simulated Administration}

At the start of the simulation session, individual participants entered a room containing a multipurpose manikin (model MW25 11404-000, Kyoto Kagaku Co., Ltd) lying supine on the floor, an investigator, three evaluators (physician, nurse, and/or pharmacist), a timekeeper, and a table with glucagon administration equipment. For NG administration by caregivers, the table contained an NG device identical to the commercially representative product but without drug powder, a Humalog ${ }^{\circledR}$ MirioPen $^{\circledR}\left(\right.$ KwikPen $\left.^{\circledR}\right)$ (Eli Lilly Japan K.K.), an Insulin Glargine BS Injection [Lilly] ${ }^{\circledR}$ cartridge 
(Eli Lilly Japan K.K.), and a 32-gauge needle (BD Micro-Fine $^{\text {TM }}$ Plus). For IMG administration by caregivers, the table contained Glucagon G Novo ${ }^{\circledR} 1 \mathrm{mg}$ (Novo Nordisk Pharma Ltd.), a Humalog ${ }^{\circledR}$ MirioPen ${ }^{\circledR}\left(\right.$ KwikPen $\left.^{\circledR}\right)$, an Insulin Glargine BS Injection [Lilly] cartridge, a 32-gauge needle, a $2.5-\mathrm{mL}$ disposable syringe with 23-gauge needle (Terumo syringe $2.5 \mathrm{~mL}$ with $23 \mathrm{G}$ ), and alcohol swabs. For both caregiver scenarios, the insulin products were provided as decoys. For NG administration by third parties, the table contained only an NG device identical to the commercially representative product but without drug powder.

At the start of the simulated administration, the participant was told that the patient (i.e., the manikin) was unconscious as a result of severe hypoglycemia and required emergency glucagon treatment. Immediately after the simulation started, the timekeeper initiated loud sounds (ambulance siren and phone ringtone of approx. $80 \mathrm{~dB}$ ) to add stress to the participant, as in a real emergency. Participants could not ask for instructions on how to administer glucagon during the simulation nor were printed instructions available; however, the NG package illustration was available. The simulation ended when the participant completed or abandoned glucagon administration, or if a caregiver administered insulin instead of glucagon, or if $15 \mathrm{~min}$ had elapsed.

\section{Outcome Measures}

The primary study end point was the percentage of participants with successful administration of glucagon by caregivers using each method. Administration was considered successful if all critical steps (see Table S1 in the electronic supplementary material) were completed correctly (as judged by the majority of evaluators) and a full dose $(100 \%$ for NG device, at least 90\% for IMG) was administered. Administration was considered partial (IMG only) if all critical steps were completed correctly, but a partial dose (greater than 0\% and less than 90\%) was administered. Administration was considered failed if not all critical steps were completed correctly, or if the dose was $0 \%$, or if insulin was administered instead of glucagon.

Participant demographics and background (including patient background for caregivers, with permission from the patients) were obtained by interview at visit 1 . Outcome measures included the time needed to complete administration (time from being asked to start simulated administration to the end of simulation) and administration status (successful, partial, failed). For NG administration, in accordance with the device's characteristics, the administered dose was considered to be $100 \%$ if the plunger was pushed in until the green line was no longer visible; otherwise, the dose was considered to be $0 \%$. For IMG administration, the relative dose (i.e., percentage reduction in weight from before administration to after administration, based on the sum of the full dose of $1000 \mathrm{mg}$, glucagon vial, solution vial, and syringe with needle) was calculated; doses greater than $0 \%$ and less than $90 \%$ were considered partial doses, and doses of at least 90\% were considered full doses. The time needed to complete administration was measured as the time from when the participant was told to start administration to the time when the participant removed the NG device or needle from the manikin. If the time exceeded $15 \mathrm{~min}$, or if the participant administered insulin or abandoned glucagon administration, the administration was considered incomplete.

After each simulation session, participants were given a satisfaction questionnaire consisting of six questions regarding the specific glucagon device just used (see Table S2 in the electronic supplementary material). Each question included the options "strongly agree", "agree", "disagree", or "strongly disagree" as answers. In addition, at the end of the second simulation, caregivers were given a user-preference questionnaire consisting of eight questions regarding their preferences between the NG and IMG administration methods (see Table S3 in the electronic supplementary material).

\section{Statistical Analysis}

We aimed to enroll 20 caregivers (10 with a relative with T1D, 10 with a relative with T2D) to ensure that at least 16 caregivers completed the study. Assuming successful administration in $94 \%$ and $13 \%$ of attempts for NG and IMG, respectively [15], this sample size would provide greater than $99 \%$ power to detect a statistically 
significant difference between NG and IMG with a two-sided alpha of 0.05. For balance, we aimed to enroll 20 participants in the third-party cohort.

Analyses were conducted on all caregivers who were randomized and completed simulated administrations with both the NG device and IMG, and on all third parties who completed the simulated NG administration. Comparisons between administration methods within the caregiver cohort were analyzed using the McNemar test (exact method) for categorical outcomes and the Wilcoxon signed-rank test for continuous outcomes. Comparisons between the caregiver and third-party cohorts for the NG device were analyzed using the Fisher's exact test for categorical outcomes and the Wilcoxon rank sum test for continuous outcomes. Time-to-event analyses were conducted using the Kaplan-Meier method; participants who could not administer a dose or failed the simulated administration were censored. Time to complete administration is reported as mean, standard deviation (SD), median, minimum, and maximum in minutes and in seconds for administration attempts considered successful or partial. Participants who could not administer a dose or failed the simulated administration were treated as censored at the maximum allowable time $(15 \mathrm{~min})$. The percentage of participants completing successful/partial administration in less than $1 \mathrm{~min}, 1 \mathrm{~min}$ to less than $2 \mathrm{~min}, 2 \mathrm{~min}$ to less than $3 \mathrm{~min}, 3 \mathrm{~min}$ to less than $5 \mathrm{~min}$, and $5 \mathrm{~min}$ to less than $10 \mathrm{~min}$ is also reported for each cohort and device. Satisfaction and user-preference questionnaire data are reported as the percentage of participants giving each possible answer for each cohort and device, as applicable.

\section{RESULTS}

\section{Participant Disposition and Demographic and Baseline Clinical Characteristics}

Twenty participants were enrolled in both the caregiver and third-party cohorts (Fig. 2). One caregiver randomized to administer NG first (group 1) withdrew by participant decision after visit 1 but before the first simulation. All other participants completed the study.
In the caregiver cohort, participant characteristics in the two randomized groups were well balanced (Table 1). Most caregivers were women $(80 \%)$, were the spouse of a patient with diabetes $(60 \%)$, and had not had previous experience with severe hypoglycemia in the patient $(85 \%)$. In contrast, there were equal numbers of men and women in the third-party cohort (Table 1).

\section{Percentage of Successful Simulated Glucagon Administrations}

Within the caregiver cohort, the percentage of successful glucagon administrations was significantly greater with NG than with IMG $(89.5 \%$ vs $26.3 \%, P<0.001$; Table 2$)$. In the third-party cohort, 95\% of NG administrations were successful; this percentage did not differ from that in the caregiver cohort. The percentage of failed administrations with IMG (15.8\%) was numerically greater compared with NG (10.5\%) in the caregiver cohort, and compared with NG in the third-party cohort (5.0\%).

The reasons that caregivers failed simulated administration were (1) for the NG device, no glucagon delivered (two caregivers who did not push the plunger completely), and (2) for the IMG device, gave up (two caregivers), incompletion of critical steps (three caregivers), and no glucagon delivered (two caregivers). Three caregivers started to prepare insulin for injection instead of glucagon, but none of the caregivers administered insulin during the simulation. No caregivers or third-party participants started to prepare insulin for administration with the NG device. No caregivers attempted to administer insulin to the manikin for either method.

\section{Time to Administer Glucagon}

Within the caregiver cohort, the time required to complete successful or partial (IMG only) glucagon administration was significantly shorter $(P<0.001)$ with the NG device (mean $23.9 \mathrm{~s}$ ) than with IMG (mean $207.3 \mathrm{~s}$; Table 3 and Fig. 3a). Third parties required significantly longer than caregivers for NG administration 


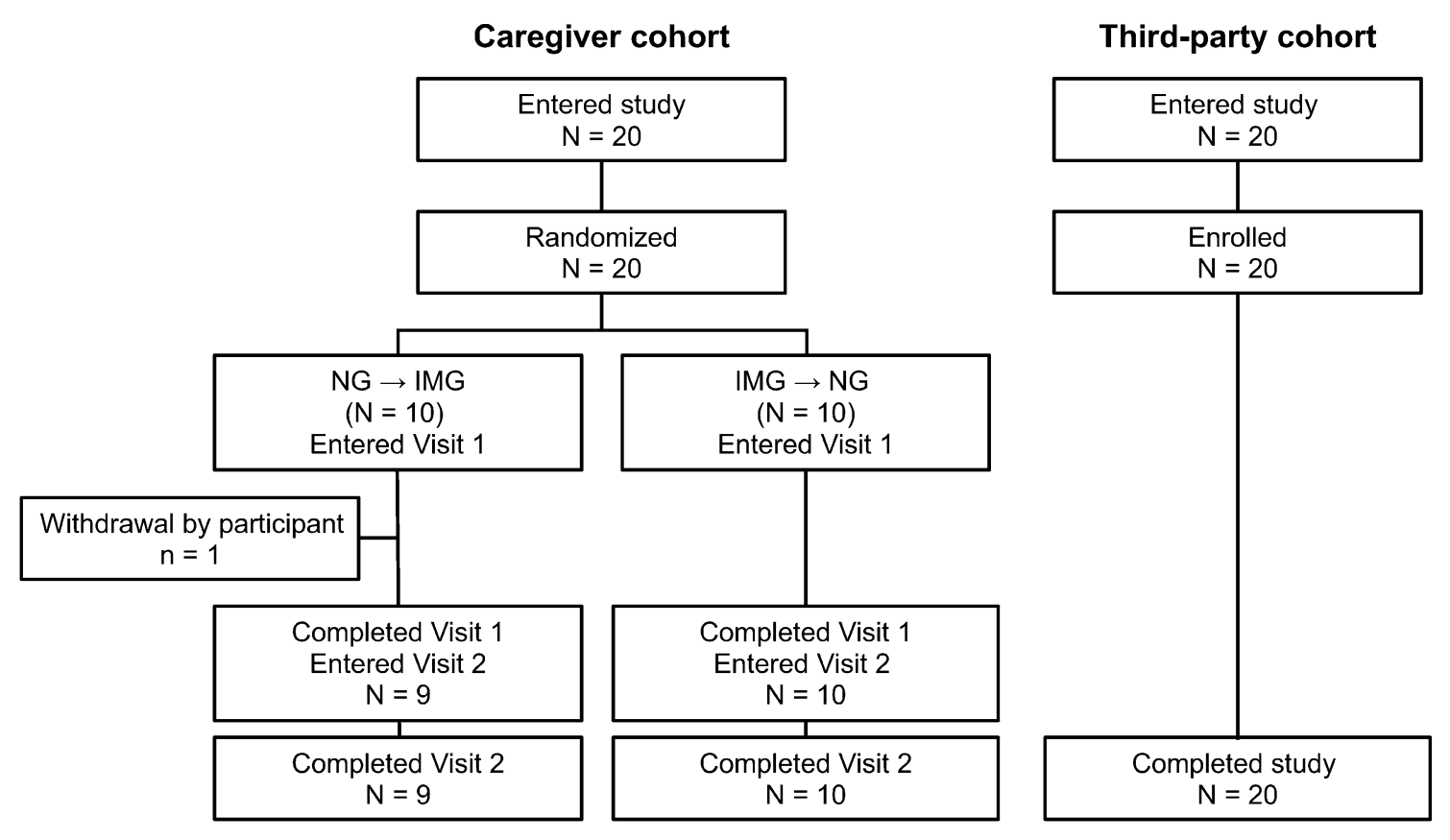

Fig. 2 Disposition of participants in the caregiver and third-party cohorts. $N G$ nasal glucagon, $I M G$ intramuscular glucagon

(mean 55.5 vs 23.9 s, respectively; Table 3 and Fig. $3 \mathrm{~b}$ ). All caregivers and $63.2 \%$ of third parties completed NG administration within $1 \mathrm{~min}$ (Fig. 4). In contrast, no caregivers completed IMG administration within $1 \mathrm{~min}$, and most (56.3\%) required 3-5 min.

\section{Device Preference}

All caregivers and most third parties reported that the NG device was easy to use, they were confident using the device during the simulation, and they were confident and willing to use the device in the event of a real emergency (Fig. 5). All caregivers and $70-100 \%$ of third parties answered "agree" or "strongly agree" to the five positively worded questions with respect to the NG device. Note that all caregivers and 95\% of third parties answered "disagree" or "strongly disagree" to the one negatively worded question ("The glucagon delivery method is intimidating and difficult for caregivers like me to use in case of an episode of rescue of severe hypoglycemia"). In contrast, caregivers were generally not as comfortable administering IMG, although there was some variability in the responses (Fig. 5). Only 21\% of caregivers said that they were confident they could administer IMG during a real severe hypoglycemic event. Most (70\%) caregivers found the IMG administration method "intimidating and difficult". However, $47 \%$ agreed that they would be willing to use IMG in the event of an emergency, and 58\% agreed that they would recommend their loved one have glucagon available.

When asked to directly compare the two methods of glucagon administration, caregivers strongly favored the NG device over IMG (Fig. 6). More than $80 \%$ of caregivers reported that they preferred the NG device in terms of ease, confidence, and willingness in using the device. In contrast, more than $70 \%$ of caregivers thought that IMG administration was unacceptable for successful rescue during a severe hypoglycemic event.

\section{DISCUSSION}

This simulated user experience study confirms and extends previous findings [15] that nasal administration of glucagon is easier, quicker, and had a higher success rate than intramuscular administration. This study is the first to compare NG and IMG administration by 
Table 1 Participant demographics and characteristics

\begin{tabular}{|c|c|c|c|c|}
\hline & \multicolumn{3}{|l|}{ Caregiver cohort } & \multirow{2}{*}{$\begin{array}{l}\text { Third-party cohort } \\
(N=20)\end{array}$} \\
\hline & Group $1^{a}(N=10)$ & Group $2^{b}(N=10)$ & Total $(N=20)$ & \\
\hline \multicolumn{5}{|l|}{ Both cohorts } \\
\hline \multicolumn{5}{|l|}{ Sex, $n(\%)$} \\
\hline Female & $8(80.0)$ & $8(80.0)$ & $16(80.0)$ & $10(50.0)$ \\
\hline Male & $2(20.0)$ & $2(20.0)$ & $4(20.0)$ & $10(50.0)$ \\
\hline \multicolumn{5}{|l|}{ Age, years } \\
\hline Mean (SD) & $49.5(9.91)$ & $47.3(14.94)$ & $48.4(12.39)$ & $44.3(14.90)$ \\
\hline Median (min, max) & $50.0(34,66)$ & $52.0(23,64)$ & $50.0(23,66)$ & $46.0(21,67)$ \\
\hline \multicolumn{5}{|l|}{ Caregivers only } \\
\hline \multicolumn{5}{|c|}{ Relationship with the patient, $n$ (\%) } \\
\hline Spouse & $6(60.0)$ & $6(60.0)$ & $12(60.0)$ & - \\
\hline Child & $4(40.0)$ & $3(30.0)$ & $7(35.0)$ & - \\
\hline Mother & $0(0.0)$ & $1(10.0)$ & $1(5.0)$ & - \\
\hline \multicolumn{5}{|c|}{ Patient diabetes type, $n(\%)$} \\
\hline Type 1 & $5(50.0)$ & $5(50.0)$ & $10(50.0)$ & - \\
\hline Type 2 & $5(50.0)$ & $5(50.0)$ & $10(50.0)$ & - \\
\hline \multicolumn{5}{|c|}{ Patient diabetes duration, years } \\
\hline Mean (SD) & $15.5(14.39)$ & $12.2(7.86)$ & $13.9(11.41)$ & - \\
\hline Median (min, max) & $14.0(1,50)$ & $11.5(1,22)$ & $13.0(1,50)$ & - \\
\hline \multicolumn{5}{|c|}{ Experience with severe hypoglycemia in a cohabiting patient, $n(\%)$} \\
\hline No & $8(80.0)$ & $9(90.0)$ & $17(85.0)$ & - \\
\hline Yes & $2(20.0)$ & $1(10.0)$ & $3(15.0)$ & - \\
\hline
\end{tabular}

max maximum, min minimum, $S D$ standard deviation

a Group 1: nasal glucagon $\rightarrow$ intramuscular glucagon

b Group 2: intramuscular glucagon $\rightarrow$ nasal glucagon

Japanese caregivers and third parties. Most caregivers and third parties could use the NG device correctly and reported that they were confident in its use. These results suggest that the availability of a ready-to-use form of glucagon (i.e., NG device) in Japan may increase the number of patients with diabetes who keep glucagon on hand in case of emergency [9].

Both the speed and accuracy of glucagon administration were significantly greater with the NG device than with IMG. The high success rate with NG administration observed in this study is consistent with the previous Canadian simulation study, in which 15 of 16 (94\%) caregivers and 14 of 15 (93\%) third parties delivered a full dose of glucagon with the NG device [15]. In contrast, only a quarter of IMG administration attempts by caregivers were successful in our study, and many caregivers administered only a partial dose by this route. 
Table 2 Percentage of participants with successful, partial, or failed simulated administration

\begin{tabular}{llll}
\hline & \multicolumn{2}{l}{ Caregiver cohort } & Third-party cohort \\
\cline { 2 - 4 } & NG $(\boldsymbol{N}=\mathbf{1 9})$ & IMG $(\boldsymbol{N}=\mathbf{1 9})$ & NG $(\boldsymbol{N}=\mathbf{2 0})$ \\
\hline Administration outcome, $n(\%)$ & $17(89.5)$ & $5(26.3)$ & $19(95.0)$ \\
Successful & NA & $11(57.9)$ & NA \\
Partial & $2(10.5)$ & $3(15.8)$ & $1(5.0)$ \\
Failed & & $<0.001^{\mathrm{a}}$ & $0.605^{\mathrm{b}}$ \\
$P$ value vs caregiver NG & & & \\
\hline
\end{tabular}

$I M G$ intramuscular glucagon, $N A$ not applicable, $N G$ nasal glucagon

a McNemar test (exact method)

b Fisher's exact test

Table 3 Time required to complete administration (successful or partial)

\begin{tabular}{llll}
\hline & \multicolumn{2}{l}{ Caregiver cohort } & Third-party cohort \\
\cline { 2 - 4 } & NG $(\boldsymbol{N}=\mathbf{1 9})$ & IMG $(\boldsymbol{N}=\mathbf{1 9})$ & NG $(\boldsymbol{N}=\mathbf{2 0})$ \\
\hline Time to administer glucagon (min) & 17 & 16 & 19 \\
$n$ & $0.399(0.1015)$ & $3.455(1.2232)$ & $0.925(0.5259)$ \\
Mean (SD) & $0.383(0.23,0.62)$ & $3.350(1.68,6.02)$ & $0.783(0.33,2.50)$ \\
Median (min, max) & & \\
Time to administer glucagon $(s)$ & 17 & 16 & 19 \\
$n$ & $23.9(6.09)$ & $207.3(73.39)$ & $55.5(31.56)$ \\
Mean (SD) & $23.0(14,37)$ & $201.0(101,361)$ & $47.0(20,150)$ \\
Median (min, max) & $<0.001^{\mathrm{a}}$ & $<0.001^{\mathrm{b}}$ \\
$P$ value vs caregiver NG & &
\end{tabular}

$N G$ nasal glucagon, $I M G$ intramuscular glucagon, $S D$ standard deviation, max maximum, min minimum

${ }^{a}$ Wilcoxon signed-rank test

${ }^{b}$ Wilcoxon rank sum test

Interestingly, the percentages of successful and partial IM glucagon administrations in our study $(26.3 \%$ and $57.9 \%$, respectively) were higher than in the Canadian study (13\% and $38 \%$, respectively). Possible reasons for this difference include the potentially higher level of commitment of Japanese participants and the direct training from health care professionals that caregivers received, as would normally occur in Japan, whereas the Canadian study trained patients who then described the method to their caregivers. The third-party cohort was intended to simulate acquaintances or bystanders who would not typically have any training or experience administering glucagon. The training given to caregivers in our study, and the lack of training given to third parties, was intended to simulate these real-life circumstances.

In this study, all caregivers and most third parties could successfully complete NG administration in less than a minute, whereas IMG 
a Caregivers: NG device vs IMG

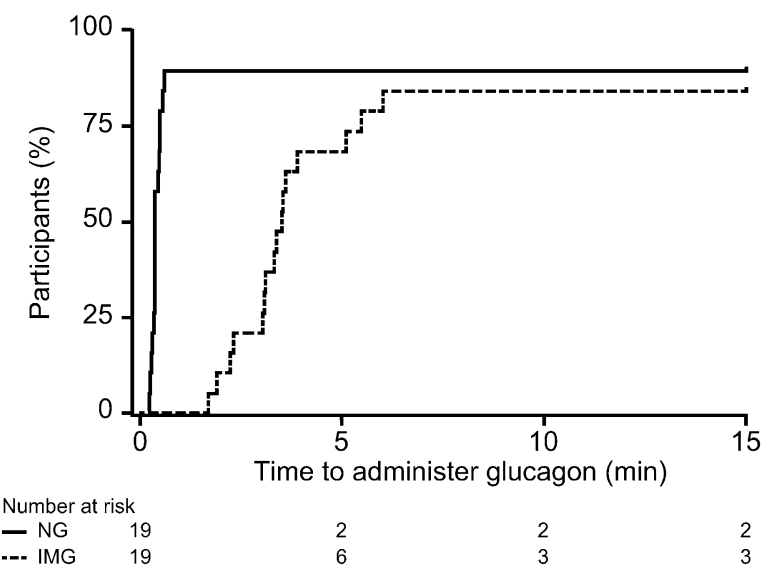

b NG device: Caregivers vs third parties

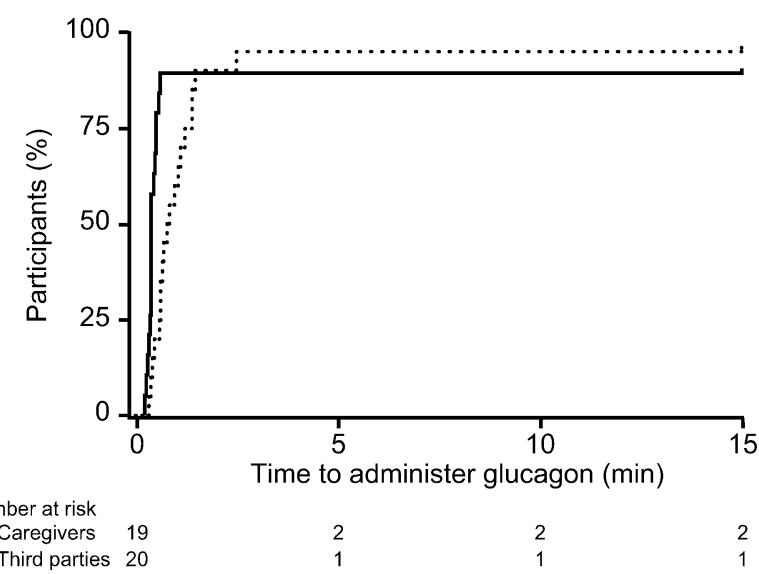

Fig. 3 Time-to-event analyses for the time needed to complete successful and partial simulated administrations. $N G$ nasal glucagon, $I M G$ intramuscular glucagon

Caregiver IMG

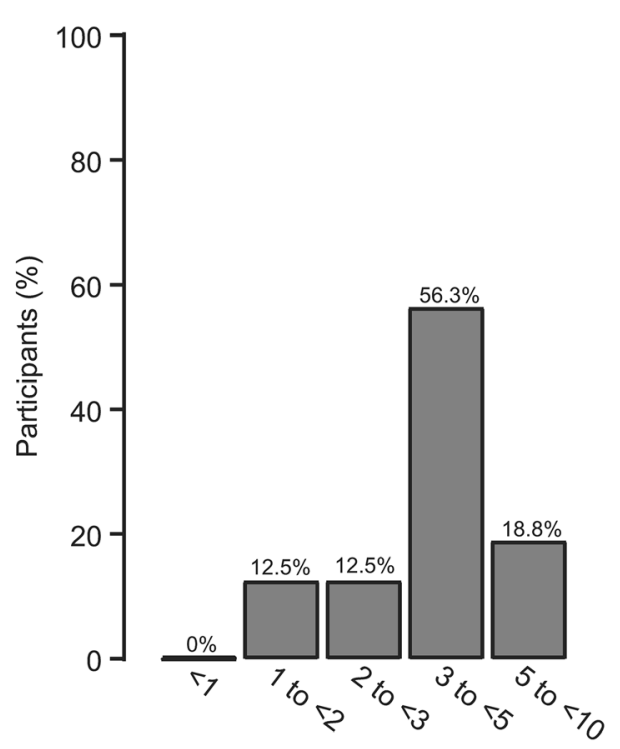

Caregiver NG

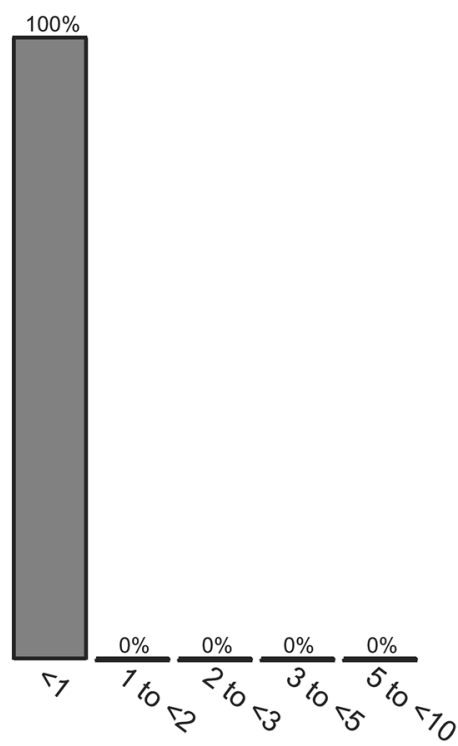

Time to administer glucagon (min)
Third-party NG

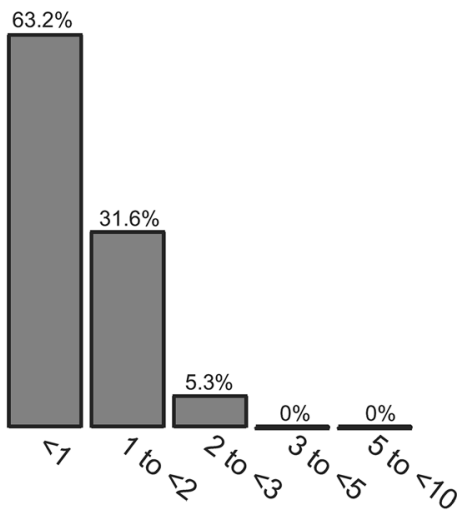

Fig. 4 Distribution of time required to administer glucagon (successful and partial administration only). $N G$ nasal glucagon, $I M G$ intramuscular glucagon

administration by caregivers took an average of about $3.5 \mathrm{~min}$. In the Canadian study, the average administration times required by caregivers for both NG (16 s) and IMG (1.89 min) were shorter than the times observed in our study [15]. The longer administration times observed in our study for the NG device are likely related to the shrink-wrap packaging and, for IMG, is likely related to the additional steps required to assemble the separate components for injection, as opposed to the glucagon kit used in the Canadian study [15]. Notably, during the IMG simulation, three caregivers started to prepare insulin for injection, although none 
1. It was easy for me to use the glucagon.

2. I am confident I used the glucagon properly.
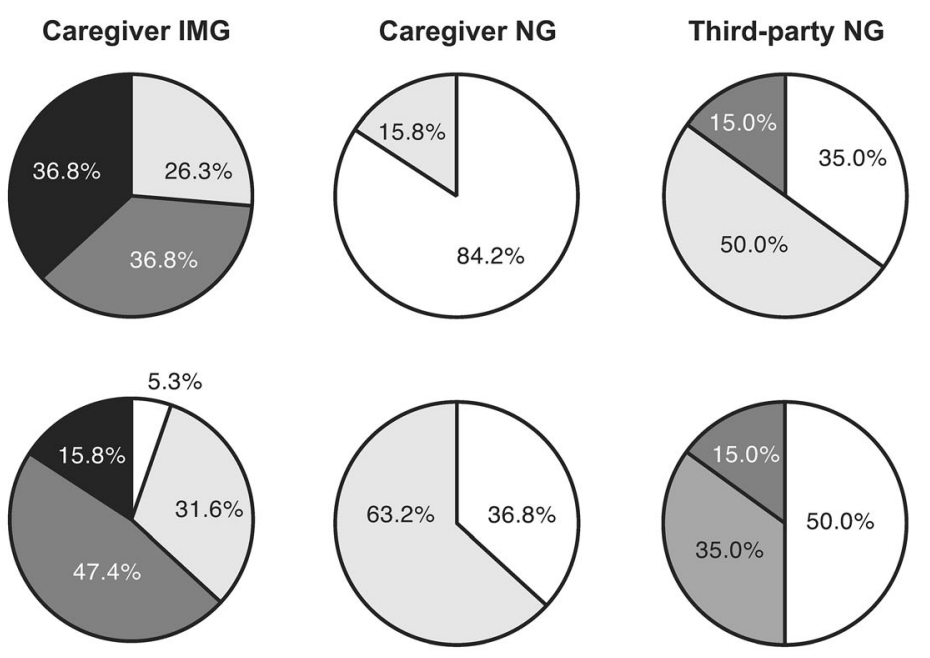

3. I am confident I would have used the glucagon properly if the mannequin was a real person, unconscious or seizing, suffering from an episode of severe hypoglycemia.
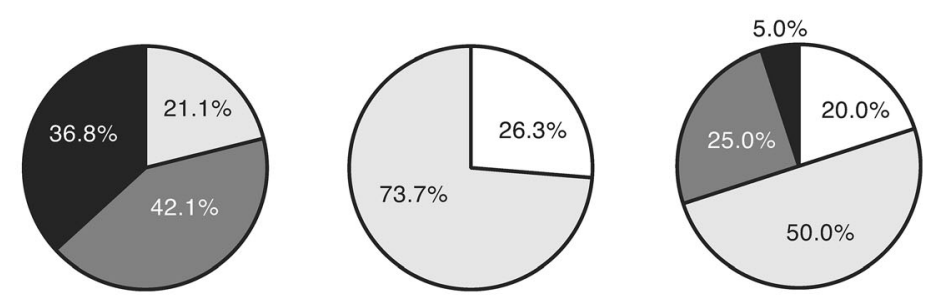

4. The glucagon delivery method is intimidating and difficult for caregivers like me to use in case of an episode of rescue of severe hypoglycemia. ${ }^{*}$
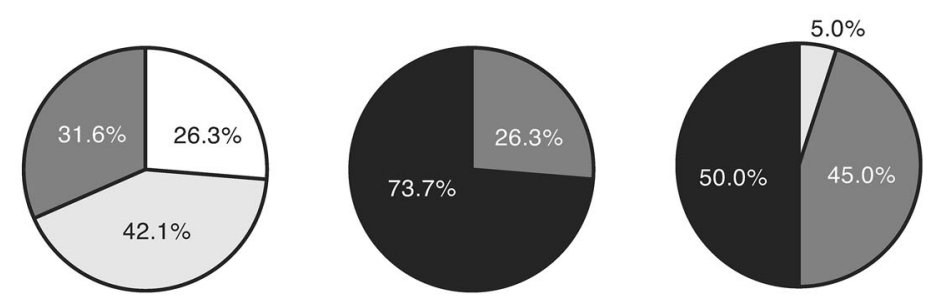

5. I am willing to use the glucagon in case the patient becomes unconscious due to severe hypoglycemia.
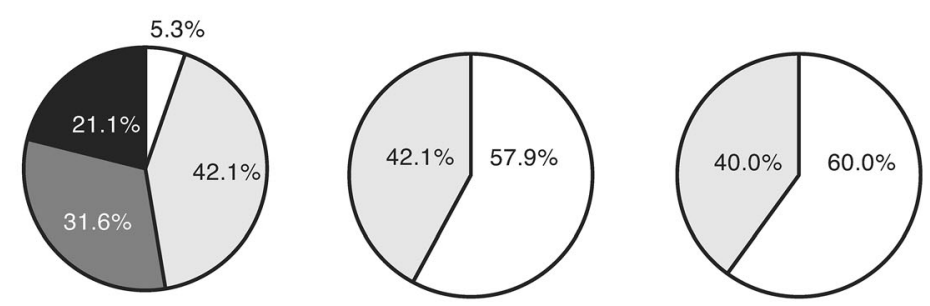

6. I would recommend my loved one or friend with diabetes to prepare the glucagon so that I can treat them in case of an episode of severe hypoglycemia.
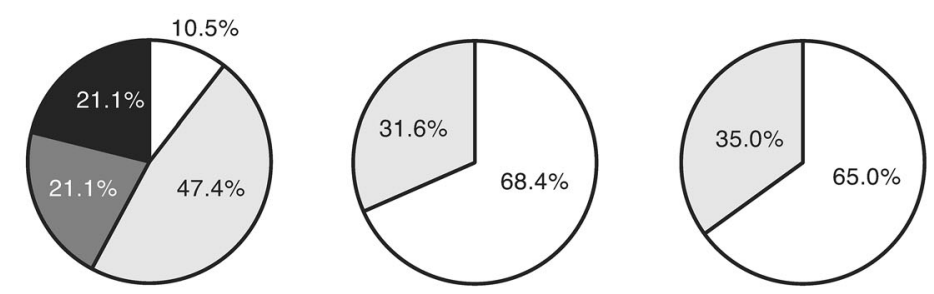

\section{Strongly agree}

Agree

Disagree

Strongly disagree

*Note that Question 4 is a negatively worded question. 
4 Fig. 5 User satisfaction with the NG and IMG administration methods. Shown are the percentages of participants who responded "strongly agree", "agree", "disagree", or "strongly disagree" to six questions for each cohort/ device. Note that question 4 was a negatively worded question. $N G$ nasal glucagon, $I M G$ intramuscular glucagon

actually tried to administer insulin. In the Canadian study, 5 of 16 (31\%) caregivers actually attempted to inject insulin, either instead of or in addition to glucagon [15]. Providing glucagon in a nasal delivery device would minimize the potential for confusion between glucagon and insulin during a real emergency.

Both caregivers and third parties found the NG device easy to use. Caregiver responses to the questionnaires were strongly positive about the NG device compared with the IMG administration. These results are consistent with those of the Canadian simulation study [15], as well as those of real-world studies of the NG device, in which participants indicated that the device was easy to use $[13,14]$. The possession rate for glucagon among patients with T1D in Japan is reportedly very low (15.9\%), mostly because patients do not think they will need glucagon, but also because of reluctance by family members to inject [9]. Higher glucagon possession rates have been reported in Australia (92\%) [8], Israel (60\%) [17], Croatia (45\%) [18], and the USA (49\%) [19]; this difference may reflect the lack of a glucagon kit in Japan, the older age of patients in this study (most other studies were of pediatric patients), or cultural factors. Because the NG device is ready to use and is less intimidating than using a syringe and needle for IMG injection, availability of the NG device may help increase the number of patients who keep glucagon on hand.

Strengths of this study include the randomized, crossover study design (caregiver cohort), sufficient statistical power to assess the primary objective, inclusion of a third-party cohort to examine use of the NG device in the absence of any training, and measurement of both objective

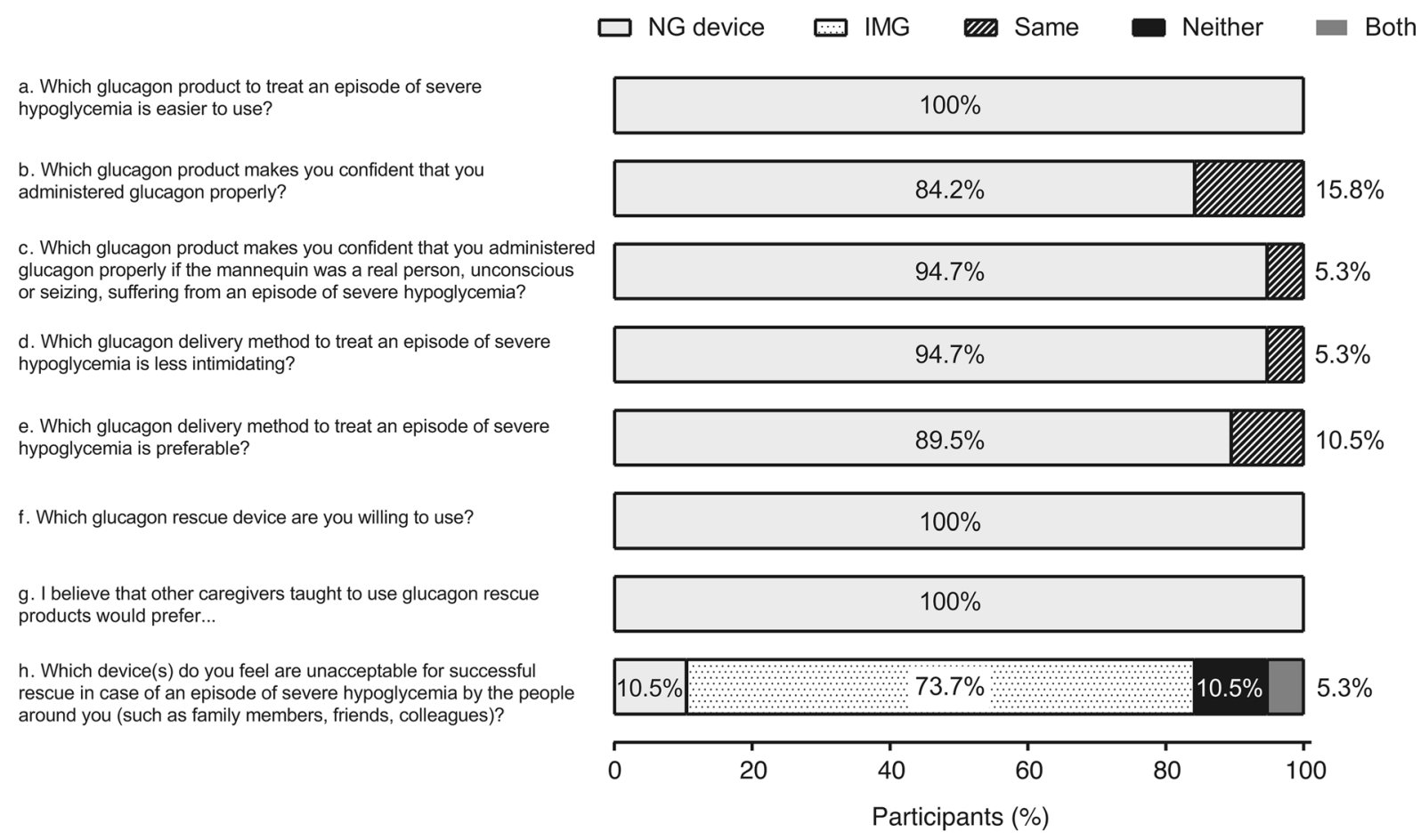

Fig. 6 Caregiver preferences for the NG or IMG administration methods. Shown are the percentages of caregivers who responded "NG device", "IMG", or "same" for

questions a-g, and "NG device", "IM glucagon", "same", "neither", or "both" for question h. $N G$ nasal glucagon, $I M G$ intramuscular glucagon 
(success rate, time to completion) and subjective (satisfaction and user-preference) outcomes. However, it should be acknowledged that this was a simulation study that could not exactly replicate a real-life emergency, including the stress and fear of the situation, and where the time elapsed between training and administration of glucagon in a real-life emergency is likely to be longer. Further, as drug administration by a third party is prohibited by the Medical Practitioners' Act in Japan, we did not test the use of IMG by third parties. Under this condition, it was not appropriate to conduct a simulated administration study using IMG in third parties without training, considering the risk of accidental needlestick injury. In contrast, because NG can be administered easily without apparent safety risks to the user, we could evaluate the percentage of successful NG administration by third parties who had no training. The study was also open-label out of necessity. All participants were adults, and most caregivers looked after an adult patient with diabetes; the results may be different for caregivers who look after a child or for adolescents who might administer glucagon. Finally, the results with Japanese participants may not be applicable to people from other countries, particularly because the glucagon injection kit available in most other countries is not available in Japan.

\section{CONCLUSION}

This simulation study confirmed that administration of glucagon using a nasal delivery device was quicker, easier, and successful more frequently than administration by intramuscular injection in Japan, where the glucagon injection kit is not available. The availability of an NG delivery device would likely increase the willingness of patients and their caregivers to keep glucagon with them and administer it in the event of severe hypoglycemia.

\section{ACKNOWLEDGEMENTS}

The authors would like to thank all study participants, and physicians and staff at each investigational site for their contributions to this study.

Funding. This study was sponsored by Eli Lilly Japan K.K. (Kobe, Japan), developer of a nasal glucagon device. Eli Lilly Japan K.K. was involved in the study design, data collection, data analysis, and preparation of the manuscript, and funded the costs of Open Access. All authors had full access to all of the data in this study and take complete responsibility for the integrity of the data and accuracy of the data analysis.

Medical Writing and/or Editorial Assistance. Medical writing assistance was provided by Rebecca Lew, PhD, CMPP, and Luke Carey, PhD, CMPP of ProScribe-Envision Pharma Group, and was funded by Eli Lilly Japan K.K. ProScribe's services complied with international guidelines for Good Publication Practice (GPP3).

Authorship. All named authors meet the International Committee of Medical Journal Editors (ICMJE) criteria for authorship for this article, take responsibility for the integrity of the work as a whole, and have given their approval for this version to be published.

Authorship Contributions. Toshihiko Aranishi, Yasushi Takita and Shuyu Zhang were involved in the study design; Yukiko Nagai and Rimei Nishimura were involved in data collection; Yasushi Takita and Shuyu Zhang conducted the statistical analysis; and all authors were involved in data interpretation.

Disclosures. Toshihiko Aranishi is an employee and shareholder of Eli Lilly Japan K.K. or Eli Lilly and Company. Yukiko Nagai is an employee and shareholder of Eli Lilly Japan K.K. or Eli Lilly and Company. Yasushi Takita is an employee and shareholder of Eli Lilly Japan K.K. or Eli Lilly and Company. Shuyu Zhang is an employee and shareholder of Eli Lilly and Company. Rimei Nishimura has received speaker honoraria from Astellas Pharma, Nippon Boehringer Ingelheim, Eli Lilly Japan K.K., Kissei Pharmaceutical, Medtronic Japan, MSD, Novartis Pharma K.K., Novo Nordisk Pharma, 
Sanofi K.K., and Takeda Pharmaceutical, and contract research fees for collaborative research with the Japan Diabetes Foundation.

Compliance with Ethics Guidelines. The protocol was approved by the ethics review committee of the study site (PS Clinic), and the study was conducted in accordance with the principles of the Declaration of Helsinki, Ethical Guidelines for Medical and Health Research Involving Human Subjects in Japan [16], and all applicable laws and regulations. As this was a non-interventional trial, submission to a clinical trial registry was not required. All participants provided informed consent to participate before the start of the study.

Data Availability. Lilly provides access to all individual participant data collected during the trial, after anonymization, with the exception of pharmacokinetic or genetic data. Data are available to request 6 months after the indication studied has been approved in the USA and EU and after primary publication acceptance, whichever is later. No expiration date of data requests is currently set once data are made available. Access is provided after a proposal has been approved by an independent review committee identified for this purpose and after receipt of a signed data sharing agreement. Data and documents, including the study protocol, statistical analysis plan, clinical study report, and blank or annotated case report forms, will be provided in a secure data sharing environment. For details on submitting a request, see the instructions provided at www.vivli.org.

Open Access. This article is distributed under the terms of the Creative Commons Attribution-NonCommercial 4.0 International License (http://creativecommons.org/licenses/ by-nc/4.0/), which permits any noncommercial use, distribution, and reproduction in any medium, provided you give appropriate credit to the original author(s) and the source, provide a link to the Creative Commons license, and indicate if changes were made.

\section{REFERENCES}

1. American Diabetes Association. 6. Glycemic targets: standards of medical care in diabetes-2019. Diabetes Care. 2019;42:S61-70.

2. Kalra S, Mukherjee JJ, Venkataraman S, et al. Hypoglycemia: the neglected complication. Indian J Endocrinol Metab. 2013;17:819-34.

3. Villani M, de Courten B, Zoungas S. Emergency treatment of hypoglycaemia: a guideline and evidence review. Diabet Med. 2017;34:1205-11.

4. Fidler C, Elmelund Christensen T, Gillard S. Hypoglycemia: an overview of fear of hypoglycemia, quality-of-life, and impact on costs. J Med Econ. 2011;14:646-55.

5. Nefs G, Pouwer F, Holt RIG, et al. Correlates and outcomes of worries about hypoglycemia in family members of adults with diabetes: the second Diabetes Attitudes, Wishes and Needs (DAWN2) study. J Psychosom Res. 2016;89:69-77.

6. Polonsky WH, Fisher L, Hessler D, Johnson N. Emotional distress in the partners of type 1 diabetes adults: worries about hypoglycemia and other key concerns. Diabetes Technol Ther. 2016;18:292-7.

7. Kedia N. Treatment of severe diabetic hypoglycemia with glucagon: an underutilized therapeutic approach. Diabetes Metab Syndr Obes. 2011;4:337-46.

8. Harris G, Diment A, Sulway M, Wilkinson M. Glucagon administration-underevaluated and undertaught. Pract Diabetes Int. 2001;18:22-5.

9. Murata T, Okazaki K, Yanagisawa K, et al. Glucagon underutilized among type 1 diabetes mellitus patients in Japan. Diabetes Technol Ther. 2013;15:748-50.

10. Boido A, Ceriani V, Pontiroli AE. Glucagon for hypoglycemic episodes in insulin-treated diabetic patients: a systematic review and meta-analysis with a comparison of glucagon with dextrose and of different glucagon formulations. Acta Diabetol. 2015;52:405-12.

11. Rickels MR, Ruedy KJ, Foster NC, et al. Intranasal glucagon for treatment of insulin-induced hypoglycemia in adults with type 1 diabetes: a randomized crossover noninferiority study. Diabetes Care. 2016;39:264-70.

12. Sherr JL, Ruedy KJ, Foster NC, et al. Glucagon nasal powder: a promising alternative to intramuscular glucagon in youth with type 1 diabetes. Diabetes Care. 2016;39:555-62. 
13. Deeb LC, Dulude H, Guzman CB, et al. A phase 3 multicenter, open-label, prospective study designed to evaluate the effectiveness and ease of use of nasal glucagon in the treatment of moderate and severe hypoglycemia in children and adolescents with type 1 diabetes in the home or school setting. Pediatr Diabetes. 2018;19:1007-13.

14. Seaquist ER, Dulude H, Zhang XM, et al. Prospective study evaluating the use of nasal glucagon for the treatment of moderate to severe hypoglycaemia in adults with type 1 diabetes in a real-world setting. Diabetes Obes Metab. 2018;20:1316-20.

15. Yale JF, Dulude H, Egeth $M$, et al. Faster use and fewer failures with needle-free nasal glucagon versus injectable glucagon in severe hypoglycemia rescue: a simulation study. Diabetes Technol Ther. 2017;19:423-32.

16. Ministry of Education, Culture, Sports, Science and Technology, and Ministry of Health, Labour and Welfare. Ethical guidelines for medical and health research involving human subjects [in Japanese]. 2014. http://www.mhlw.go.jp/file/06-Seisakujouhou10600000-Daijinkanboukouseikagakuka/00000694 10.pdf. Accessed 4 April 2019.

17. Yanai O, Phillip M, Harman I, Elitzur-Leiberman E, Pilpel D. IDDM patients' opinions on the use of glucagon emergency kit in severe episodes of hypoglycaemia. Pract Diabetes Int. 1997;14:40-2.

18. Vilovic M, Kurir TT, Novak A, et al. Hypoglycemia and glucagon utilization in insulin-treated diabetic patients. Exp Clin Endocrinol Diabetes. 2018. https://doi.org/10.1055/a-0741-6763.

19. Mitchell BD, He X, Sturdy IM, Cagle AP, Settles JA. Glucagon prescription patterns in patients with either type 1 or 2 diabetes with newly prescribed insulin. Endocr Pract. 2015;22:123-35. 